\title{
Disease progression and pseudoprogression following partial response with rare gingiva metastasis upon IBI308 (sintilimab) immunotherapy reflects the complexity of metastatic lung cancer: a case report
}

\author{
Haiping Jiang ${ }^{1 \#}$, Zhenguang Chen ${ }^{2}$, Yuan Gao ${ }^{1}$, Yongfeng Ding ${ }^{1}$, Qihan You ${ }^{1}$, Lei $\mathrm{Ye}^{3}$, Lifeng $\mathrm{Li}^{3}$, \\ Guifeng Liu ${ }^{3}$, Lele Song ${ }^{1,3,4} \wedge$, Nong Xu ${ }^{1}$ \\ ${ }^{1}$ Department of Medical Oncology, The First Affiliated Hospital, School of Medicine, Zhejiang University, Hangzhou, China; ${ }^{2}$ Department of \\ Surgical Oncology, The First Affiliated Hospital of Medical School of Zhejiang University, Hangzhou, China; ${ }^{3}$ HaploX Biotechnology, Auto Electric \\ Power Building, Shenzhen, China; ${ }^{4}$ Department of Radiotherapy, the 8th Medical Center of the Chinese PLA General Hospital, Beijing, China \\ \#These authors contributed equally to this work. \\ Correspondence to: Nong Xu, MD. Department of Medical Oncology, The First Affiliated Hospital, School of Medicine, Zhejiang University, 79 \\ Qingchun Rd., Hangzhou 310003, China. Email: xunonghz@163.com; Lele Song, MD, PhD. HaploX Biotechnology, 8th floor, Auto Electric Power \\ Building, Songpingshan Road, Nanshan District, Shenzhen 518055, China. Email: songlele@sina.com.
}

\begin{abstract}
The differential diagnosis of progression and pseudoprogression is one difficulty in current immunotherapy. Since the time point and criteria for pseudoprogression diagnosis are not yet unified, current diagnosis and treatment rely on imaging and pathology. Here we report a 57-year-old Chinese male presented solitary left lower lung nodule with enlarged left hilar and mediastinal lymph nodes. Bilateral adrenal nodules and bilateral parietal lobe nodules were identified. The nodules were considered malignant by CT or MRI examinations. The patient was diagnosed left lower peripheral lung cancer with left hilar and mediastinal lymph node metastasis, bilateral adrenal metastasis, and bilateral parietal lobe metastasis. Progression was observed after the first-line pemetrexed + cisplatin (PP) standard chemotherapy. Due to the identification of strong positive PD-L1 expression (90\%) in primary tissue immunohistochemistry, second-line IBI308 (sintilimab) immunotherapy was implemented. After the third cycle of immunotherapy, partial response was observed with the left lung lesion and the lung hilus and adrenal metastases, while pseudoprogression was found at the left lung and right hepatic lobe, and rare gingival progression was also identified. Palliative surgery was performed to remove the gingival metastatic lesion. The lesions of the lung, hilar and mediastinal lymph nodes and adrenal gland responded well, but the patient died due to uncontrollable progression of metastatic lesions in the brain. Whole-exome sequencing on gingival metastasis revealed pathogenic mutations in several important driver genes, including TP53, ErbB2, MET and PTEN. This study reported the coexistence of primary lesion response, pseudoprogression and progression in immunotherapy in lung cancer patient with rare gingival metastasis, and provided experience for handling mixed responses in immunotherapy.
\end{abstract}

Keywords: Lung cancer; immunotherapy; PD-1; peudoprogression; case report.

Submitted Aug 15, 2020. Accepted for publication Dec 04, 2020.

doi: $10.21037 / \mathrm{tcr}-20-2736$

View this article at: http://dx.doi.org/10.21037/tcr-20-2736

^ ORCID: 0000-0003-0296-2978. 


\section{Introduction}

Pseudoprogression refers to the enlarged lesions or new enhanced lesions by imaging assessment following radiotherapy, chemotherapy, or immunotherapy. Subsequent treatment may find that the suspected lesions are sensitive to treatment, and they are therefore not true progression or recurrence $(1,2)$. Since pseudoprogression and tumor recurrence are difficult to discriminate, it can greatly interfere with the therapeutic strategies, and even lead to erroneous treatment. Currently, differential diagnosis of pseudoprogression is dependent on the combination of pathological biopsy, imaging observations and experimental treatment $(3,4)$. However, for patients with multiple metastatic lung cancer, it is impossible to perform pathological biopsy on all lesions, and the differential response of different lesions to immunotherapy also increases the difficulty in assessment. Therefore, it may be necessary to assess the lesions separately based on their distinct features to avoid misdiagnosis of progression.

In this study, we report a case of lung adenocarcinoma with coexistence of partial response, progression and pseudoprogression, and explore the potential molecular mechanisms of rare metastasis or progression. We hope to share our diagnostic and therapeutic experience in a complicated condition involving different treatment responses. We present the following article in accordance with the CARE reporting checklist (available at http:// dx.doi.org/10.21037/tcr-20-2736).

\section{Case presentation}

Here we report a 57-year-old male patient, with a continuous smoking history of over 30 years, who was diagnosed as left lower lung peripheral lung cancer with left hilar and mediastinal lymph node metastasis, bilateral adrenal metastasis, and bilateral parietal metastasis. Needle biopsy was performed, and subsequent $\mathrm{HE}$ staining and immunohistochemistry confirmed poorly differentiated adenocarcinoma with PD-L1(+), CK(+), NapsinA(+) and TTF-1(+) (Figure 1). Quantitative polymerase chain reaction (qPCR) assay of EGFR, ALK and ROS-1 genes in primary tumor tissue showed no sensitive mutations to tyrosine kinase inhibitors (TKIs). Therefore, he was given the first-line therapy with PP regimen (Pemetrexed $900 \mathrm{mg} \mathrm{d} 1+$ Cisplatin $60 \mathrm{mg} \mathrm{d} 1-2 \mathrm{q} 3 \mathrm{w}$ ) for 4 cycles, followed by 2 cycles of maintenance chemotherapy with Pemetrexed, while progression was observed after 2 cycles of maintenance therapy. Since the primary tissue immunohistochemistry showed strong positive PDL1 expression (90\%) (Figure 1), he entered the second line immunotherapy with IBI308 (sintilimab) (Innovent Biologics Co., Ltd) in a clinical trial (NCT02937116). The left lower lung lesions and mediastinal lymph nodes became significantly smaller after 3 cycles of treatment, and were evaluated as partial response (PR), but computed tomography (CT) showed new lesions in the left lung and suspected metastases in the liver (Figure 2), with a beansized mass $(1.7 \mathrm{~cm} \times 1.5 \mathrm{~cm} \times 0.7 \mathrm{~cm})$ appeared at gingiva of the right lower jaw and symptom of gingival pain was documented in the patient. The gingival mass was removed and pathological biopsy confirmed a metastasis of poorly differentiated lung adenocarcinoma with CK (+), NapsinA $(+)$ and TTF-1 (+) (Figure 3). The whole-exome sequencing (WES) showed that the main mutations of the gingival metastasis were ErbB2 amplification (3.1 copies), MET amplification (5.1 copies), PTEN amplification (3.0 copies) and TP53del (c.675_685del, 14.96\%) with a large number of non-driver gene point mutations and insertion/deletion mutations (Tables 1,2).

Although there were suspected new lesions at left lung and liver, the primary lung and mediastinal lymph nodes showed PR after 3 cycles of IBI308 (sintilimab) treatment. The patient had a significant improvement in self-feeling and appetite. It was therefore decided to continue the medication and observation. Assessment at by the end of the $7^{\text {th }}$ cycle showed significant relief of left lung primary tumor, left lung new lesion, mediastinal lymph node, adrenal metastases, and the suspected liver lesion, and was regarded as PR (Figure 2). Meanwhile, immune pneumonia appeared around the 6th-7th cycle, and was treated with high dose methylprednisolone, and improvement was observed after 1-month treatment. Chest and abdomen CT showed stabilized primary and hepatic and adrenal lesions after 9 cycles of treatment (Figure 2), while the patient developed dizziness and nausea after 10 cycles of treatment. The magnetic resonance imaging (MRI) showed a new lesion in the right parietal lobe, which was assessed as PD. The patient reached the endpoint of clinical trial with progression-free survival (PFS) of 12.9 months (Figure 2). The new lesions on the right parietal lobe were treated with stereotactic radiosurgery, and the treatment of the thirdline therapy with Docetaxel + Bevacizumab has begun.

All procedures performed in studies involving human participants were in accordance with the ethical standards of the institutional and/or national research committee(s) and 


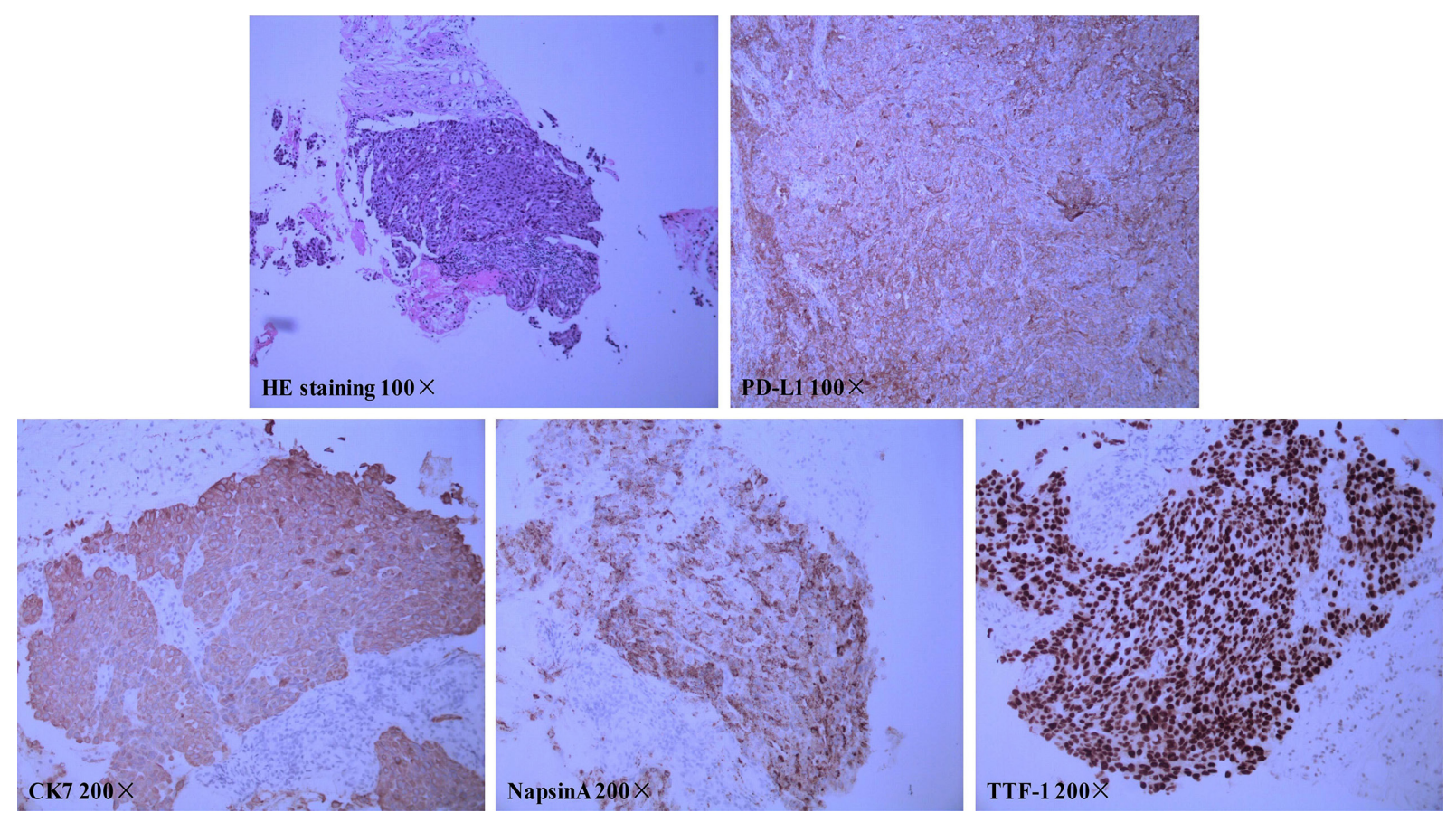

Figure 1 Pathological confirmation of left lung primary adenocarcinoma with a series of immunohistochemistry staining. Sections shown include HE staining, positive PD-L1 staining, positive CK7 staining, positive NapsinA staining and positive TTF-1 staining.

with the Helsinki Declaration (as revised in 2013). Written informed consent was obtained from the patient.

\section{Discussion}

\section{Diagnosis, therapy, and assessment in concurrence of partial response, progression and pseudoprogression}

In the case reported in this study, primary tumors, and some metastases (mediastinal lymph nodes, adrenal glands) were relieved after immunotherapy, while new metastases (gingiva) and suspicious metastases (left lung, liver) appeared. The latter showed significant response after continued treatment with the same strategy, suggesting that simultaneous target lesion relief, non-primary site progression and pseudoprogression coexisted in this case. This phenomenon is rare in current immunotherapy. The diagnosis of gingival metastasis suggests that the left lung and liver lesions may be true new metastases, but they responded well to treatment. This gives us new thinking about the definition of pseudoprogression, which may not necessarily be limited to the primary lesion or the lesions already existing at the time of diagnosis, and it may appear as new lesions after the start of immunotherapy. It was reported that biopsy can be performed to distinguish true metastasis from pseudoprogression, as immune cell infiltration and rare tumor cells were mainly found in pseudoprogression, while true progression always presented large amount of tumor cells (3-5). Therefore, if target lesions respond well while new suspected metastatic lesions appear after treatment, and the patient feel better in general condition including appetite, the treatment can be continued to confirm the diagnosis of new lesions, and responded lesions and suspected progression may be assessed separately, as discrepancy has been found between imaging and pathological assessment in some patients.

\section{WES reflected the potential molecular mechanism of rare gingival metastasis of lung adenocarcinoma}

In this case, the gingival mass was diagnosed as metastatic carcinoma, which was very rare in lung adenocarcinoma. It was reported that metastatic cancer accounted for $1 \%$ of oral tumors. The primary cancers included lung, prostate, rectal cancers in male and breast, adrenal glands, and genitalia cancers in females (6). In this study, the gingival metastasis was sequenced by WES and key driver gene variations including MET, HER2, PTEN amplification and 

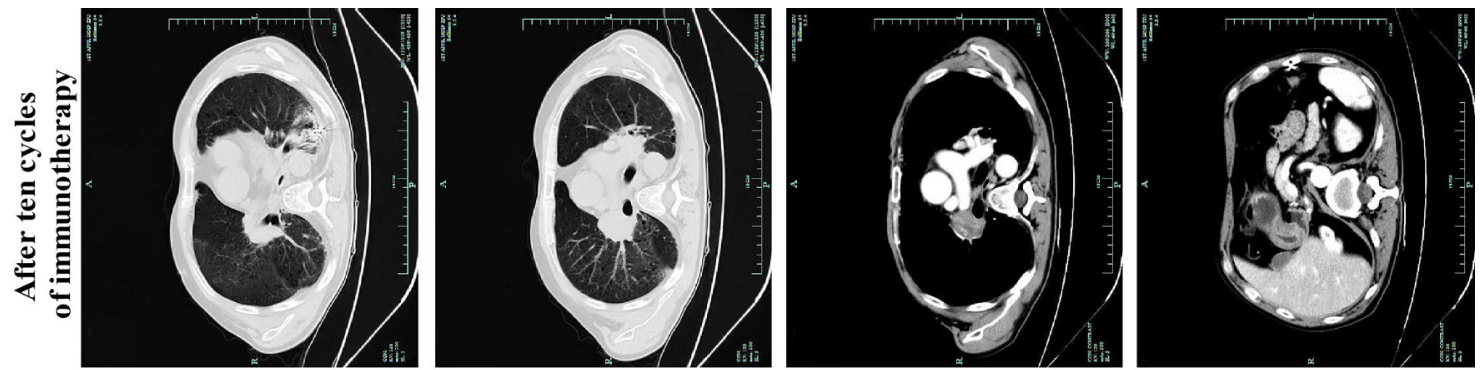

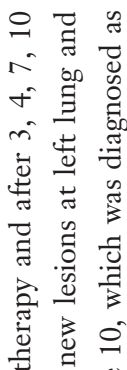
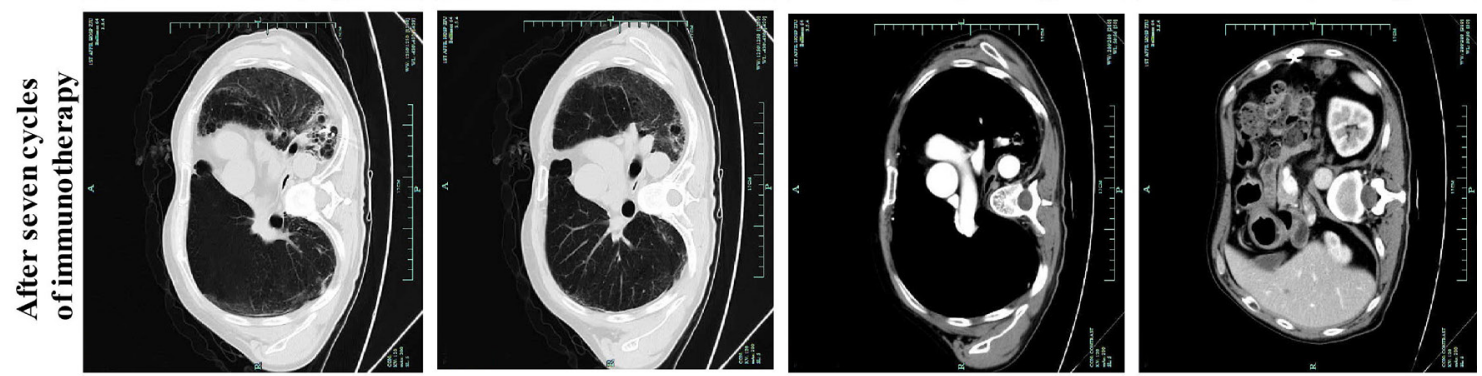

$\stackrel{0}{=} \stackrel{0}{=}$
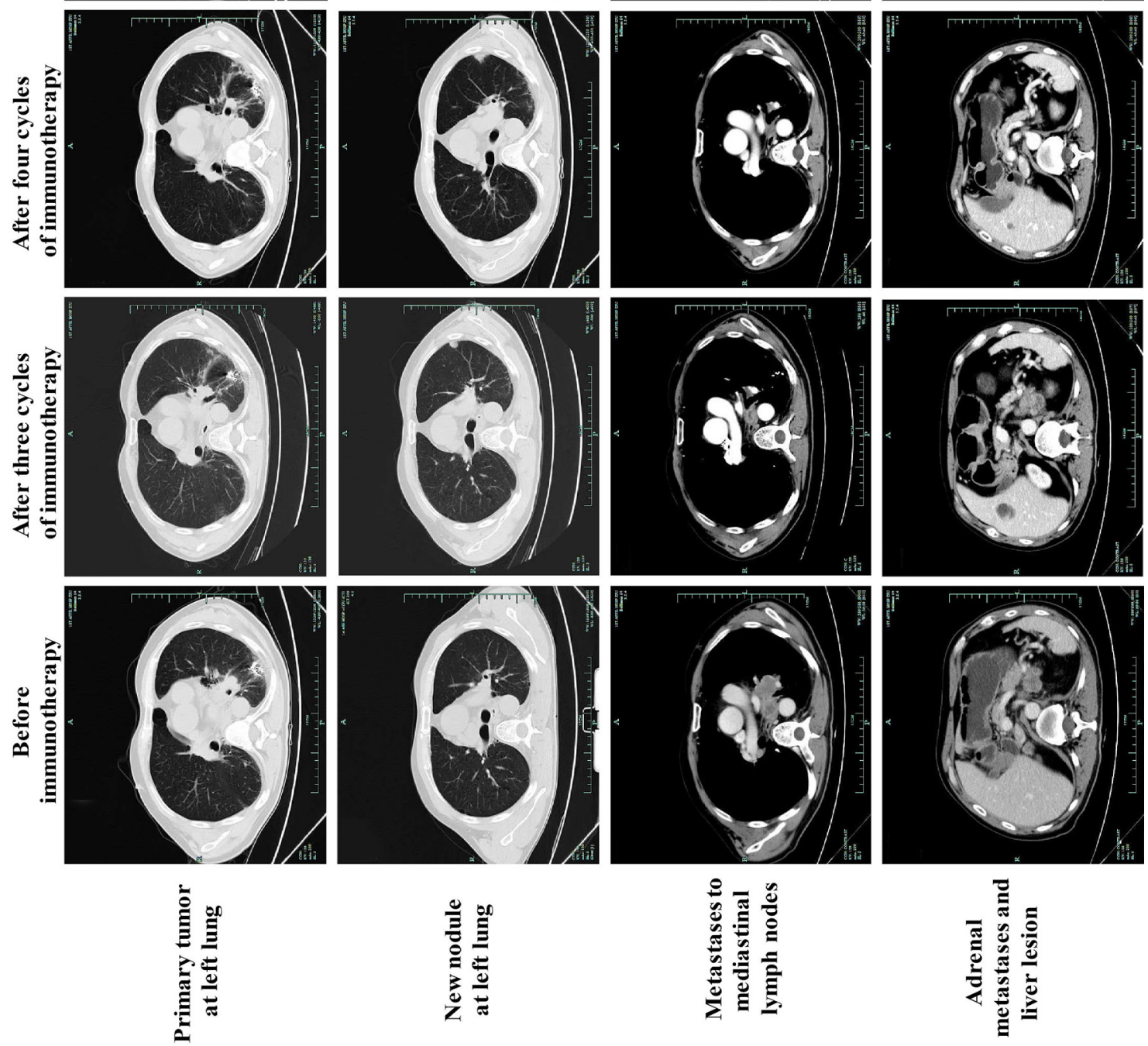

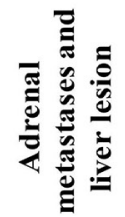

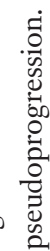



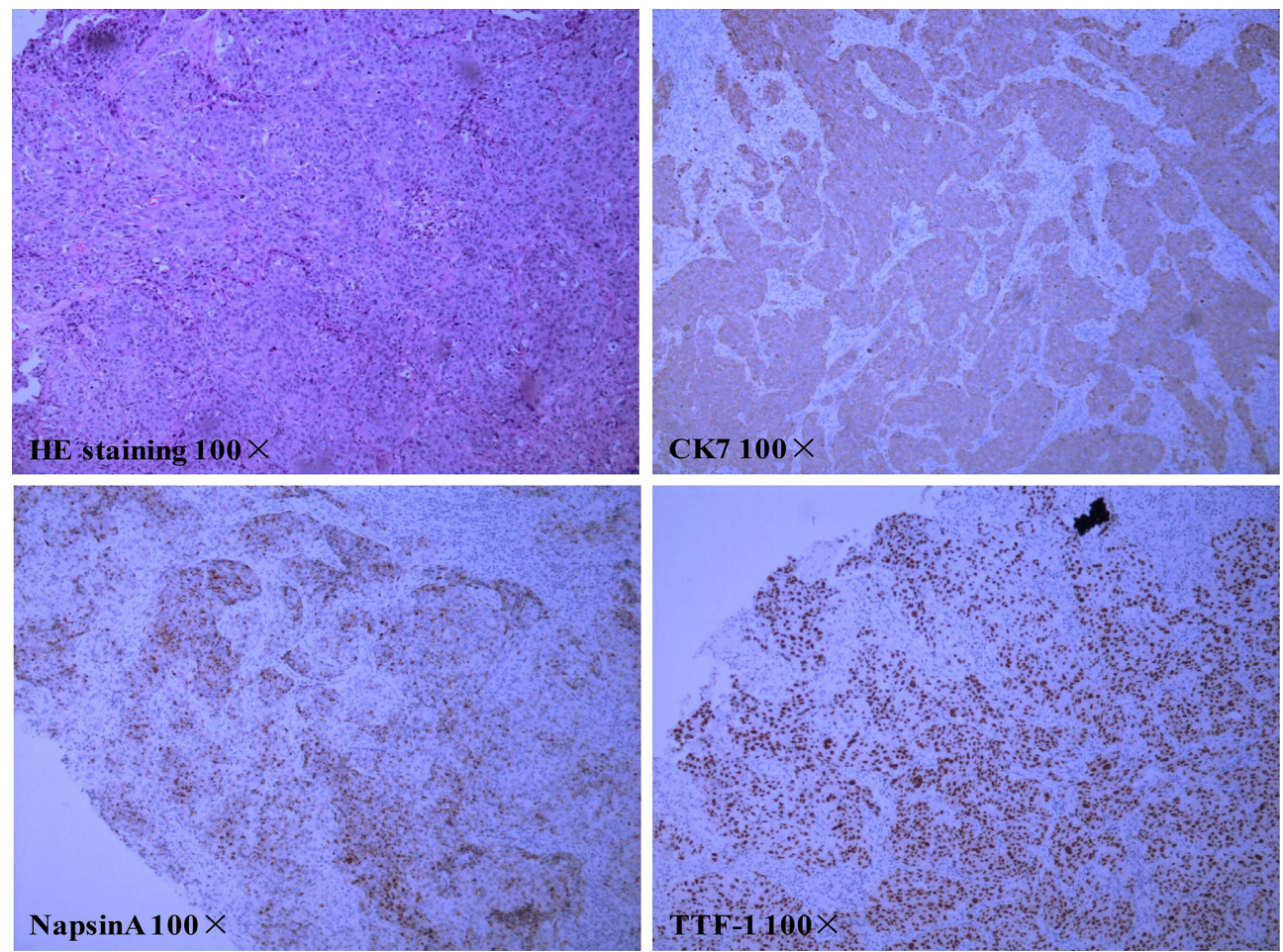

Figure 3 Pathological confirmation of adenocarcinoma of gingiva metastasis with a series of immunohistochemistry staining. Sections shown include HE staining, positive CK7 staining, positive NapsinA staining and positive TTF-1 staining.

Table 1 Main SNV and indel variations detected in tissue of gingiva metastasis

\begin{tabular}{lcccccc}
\hline Variation type & Gene name & $\begin{array}{c}\text { Chromosome } \\
\text { number }\end{array}$ & Transcript number & Exon number & Base pair change & $\begin{array}{c}\text { Amino acid } \\
\text { change }\end{array}$ Allele \\
frequency (\%)
\end{tabular}

Table 2 Main copy number variations detected in tissue of gingiva metastasis

\begin{tabular}{lclc}
\hline Variation type & Gene name & CNV change & Copy number \\
\hline CNV & ERBB2 & Amplification & 3.1 \\
& MET & Amplification & 5.1 \\
& PTEN & Amplification & 3 \\
\hline
\end{tabular}


TP53del mutations were identified. Although the complete sequencing information of the patient's primary tumor was absent, large variations of driver genes are more commonly seen in metastatic cancer than the primary cancer, which could be the driving force of metastasis (7-14).

In conclusion, we reported for the first time the coexistence of partial response with true progression and pseudoprogression, and identified the possible molecular mechanism of rare gingival metastasis. Our study provides experience and evidence for the diagnosis of pseudoprogression and therapy of multiple metastases with immunotherapy.

\section{Acknowledgments}

We thank Dr. Zili He for proof reading the manuscript as a native English-speaking expert.

Funding: This study was sponsored by Zhejiang Provincial Science and Technology Project (No. 2014C03040-2) and the National Health and Family Planning Commission Research Fund and Zhejiang Provincial Medical and Health Major Science and Technology Plan Project (No. KWJZJ-1802).

\section{Footnote}

Reporting Checklist: The authors have completed the CARE reporting checklist. Available at http://dx.doi.org/10.21037/ tcr-20-2736

Conflicts of Interest: All authors have completed the ICMJE uniform disclosure form (available at http://dx.doi. org/10.21037/tcr-20-2736). Dr. LY, Dr. LL and Dr. GL report other from HaploX Biotechnology, outside the submitted work. Dr. LS and Dr. NX report grants from Zhejiang Provincial Science and Technology Project, grants from National Health and Family Planning Commission Research Fund and Zhejiang Provincial Medical and Health Major Science and Technology Plan Project, during the conduct of the study. Dr. LS reports other from HaploX Biotechnology, Shenzhen, China, outside the submitted work. The other authors have no conflicts of interest to declare.

Ethical Statement: The authors are accountable for all aspects of the work in ensuring that questions related to the accuracy or integrity of any part of the work are appropriately investigated and resolved. All procedures performed in studies involving human participants were in accordance with the ethical standards of the institutional and/or national research committee(s) and with the Helsinki Declaration (as revised in 2013). Written informed consent for sample collection, genetic testing and publication of the case was obtained from the patient.

Open Access Statement: This is an Open Access article distributed in accordance with the Creative Commons Attribution-NonCommercial-NoDerivs 4.0 International License (CC BY-NC-ND 4.0), which permits the noncommercial replication and distribution of the article with the strict proviso that no changes or edits are made and the original work is properly cited (including links to both the formal publication through the relevant DOI and the license). See: https://creativecommons.org/licenses/by-nc-nd/4.0/.

\section{References}

1. Chiou VL, Burotto M. Pseudoprogression and ImmuneRelated Response in Solid Tumors. J Clin Oncol 2015;33:3541-3.

2. de Velasco G, Krajewski KM, Albiges L, et al. Radiologic Heterogeneity in Responses to Anti-PD-1/PD-L1 Therapy in Metastatic Renal Cell Carcinoma. Cancer Immunol Res 2016;4:12-7.

3. Ito K, Hataji O, Katsuta K, et al. "Pseudoprogression" of Pulmonary Pleomorphic Carcinoma during Nivolumab Therapy. J Thorac Oncol 2016;11:e117-9.

4. Rocha P, Hardy-Werbin M, Naranjo D, et al. CD103+CD8+ Lymphocytes Characterize the Immune Infiltration in a Case With Pseudoprogression in Squamous NSCLC. J Thorac Oncol 2018;13:e193-6.

5. Fujimoto D, Yoshioka H, Kataoka Y, et al. Pseudoprogression in Previously Treated Patients with Non-Small Cell Lung Cancer Who Received Nivolumab Monotherapy. J Thorac Oncol 2019;14:468-74.

6. Aswath N, Balakrishnan C, Shyamsundar V, et al. Oral gingival metastasis: A diagnostic dilemma. Indian J Dent Res 2017;28:344-347.

7. Jiang BY, Li YS, Guo WB, et al. Detection of Driver and Resistance Mutations in Leptomeningeal Metastases of NSCLC by Next-Generation Sequencing of Cerebrospinal Fluid Circulating Tumor Cells. Clin Cancer Res 2017;23:5480-8.

8. Qiong Z, Na WY, Bo W, et al. Alterations of a spectrum of driver genes in female Chinese patients with advanced or metastatic squamous cell carcinoma of the lung. Lung 
Cancer 2015;87:117-21.

9. Iranmanesh SM, Guo NL. Integrated DNA Copy Number and Gene Expression Regulatory Network Analysis of Non-small Cell Lung Cancer Metastasis. Cancer Inform 2014;13:13-23.

10. Kim EK, Kim KA, Lee CY, et al. The frequency and clinical impact of HER2 alterations in lung adenocarcinoma. PLoS One 2017;12:e0171280.

11. Jamal-Hanjani M, Wilson GA, McGranahan N, et al. Tracking the Evolution of Non-Small-Cell Lung Cancer. N Engl J Med 2017;376:2109-21.

Cite this article as: Jiang $\mathrm{H}$, Chen Z, Gao Y, Ding Y, You Q, Ye L, Li L, Liu G, Song L, Xu N. Disease progression and pseudoprogression following partial response with rare gingiva metastasis upon IBI308 (sintilimab) immunotherapy reflects the complexity of metastatic lung cancer: a case report. Transl Cancer Res 2021;10(1):546-552. doi: 10.21037/tcr-20-2736
12. Cheng Y, Wang S, Han L, et al. Concurrent somatic mutations in driver genes were significantly correlated with lymph node metastasis and pathological types in solid tumors. Oncotarget 2017;8:68746-57.

13. Blakely CM, Watkins TBK, Wu W, et al. Evolution and clinical impact of co-occurring genetic alterations in advanced-stage EGFR-mutant lung cancers. Nat Genet 2017;49:1693-704.

14. Reiter JG, Makohon-Moore AP, Gerold JM, et al. Minimal functional driver gene heterogeneity among untreated metastases. Science 2018;361:1033-7. 